\title{
The Importance of Recruiting a Diverse Population for Stem Cell Clinical Trials
}

\author{
Fumitaka Nagamura ${ }^{1}$
}

Published online: 4 October 2016

(C) Springer International Publishing AG 2016

\begin{abstract}
The development of stem cell therapies is evolving rapidly in parallel with advances in stem cell biology. However, many important issues remain to be addressed, ranging from the detailed molecular dissection of the therapeutic capabilities of stem cells in situ, through to the selection of both patients and sources of cells for clinical trials. Particularly pertinent to patient selection is the importance of enrolling sufficient numbers of patients in clinical trials, especially for comparative studies. Unfortunately, conducting comparative studies is often complicated by limitations on cell processing or the relative rarity of target diseases, so it is difficult to enroll sufficient numbers of patients with diverse backgrounds. Indeed, the number of patients enrolled in comparative stem cell studies is generally small, and few randomized trials have been conducted. In Japan, a new regulatory framework involving conditional and time-limited approval of regenerative products and mandatory post-marketing surveillance after early approval may help resolve the problem, because the enrollment qualification data for many patients will be collected. The translation of stem cell therapies to the clinic is still in its infancy, and stringent efforts to identify and establish suitable patient and control cohorts from diverse genetic, ethnic, and demographic backgrounds remain a high priority for the optimization of clinical trial design.
\end{abstract}

This article is part of the Topical Collection on Ethics in Stem/Progenitor Cell Therapeutics

Fumitaka Nagamura

nagamura@ims.u-tokyo.ac.jp

1 Division of Advanced Medicine Promotion, The Institute of Medical Science, The University of Tokyo, 4-6-1 Shirokanedai, Minato-ku, Tokyo 108-8639, Japan
Keywords Stem cell therapy $\cdot$ Patient selection · Efficacy evaluation $\cdot$ Patient demographics

\section{Introduction}

Clinical trials are indispensable for establishing the efficacy and safety profiles of new drug candidates prior to market approval. However, the characteristics of patients recruited for the study and control arms of clinical trials, including age, race, gender, and, more recently, molecular profiles, are often significantly skewed to a certain group. The differences in characteristics between subjects selected for clinical trials by inclusion/exclusion criteria and those treated after approval carry the potentially serious risk of compromising the efficacy and safety data [1]. The International Council for Harmonization of Technical Requirements for Pharmaceuticals for Human Use (ICH) was established to make recommendations toward achieving greater harmonization in the interpretation and application of technical guidelines and requirements for pharmaceutical product registration among Europe, Japan, and USA, thereby reducing or obviating duplication of testing carried out during the research and development of new human medicines. According to ICH guideline E5, "ICH HARMONISED TRIPARTITE GUIDELINE: ETHNIC FACTORS IN THE ACCEPTABILITY OF FOREIGN CLINICAL DATA E5 (R1)," the development of a framework for evaluating the impact of ethnic factors upon a medicine's effect, i.e., its efficacy and safety at a particular dosage and dose regimen, is an important consideration. The guideline divides ethnic factors into "intrinsic ethnic factors" and "extrinsic ethnic factors." The former includes genetic polymorphisms, age, gender, height, weight, lean body mass, body composition, and organ dysfunction. Extrinsic ethnic factors are defined as the social 
and cultural aspects of a region such as medical practice, diet, use of tobacco and alcohol, exposure to pollution and sunshine, socio-economic status, and compliance with prescribed medications. In addition, and of particular importance to studies from different regions to reduce the affects induced by ethnic differences, is clinical trial design that enables the analysis on ethnic factors and conduct practices that reduces regional difference, such as the compliance with study protocol.

Usually, clinical drug development is classified as three phases until approval. Human pharmacology and safety/ tolerability are examined in phase I studies; exploratory therapeutic effect is evaluated in phase II studies, and the efficacy of new therapies is assessed in phase III studies, which are designed to determine the effectiveness of new treatments compared to standard therapy, including best supportive care, and to evaluate the incidence and severity of adverse events in new therapies compared to standard treatments. Ultimately, products are approved for market based on the attainment of clinically equivalent or improved outcomes compared to standard therapies. For this reason, the number of patients recruited for phase III studies should be appropriate to prove the equality or superiority of new treatments based on robust biostatistical guidelines. For this to be achieved, it is not uncommon for phase III studies to involve thousands of subjects.

ICH guideline E8, "ICH HARMONISED TRIPARTITE GUIDELINE: GENERAL CONSIDERATIONS FOR CLINICAL TRIALS," emphasizes the importance of recognizing the phase of clinical trials, because the aim of trials and the validity of data may differ based on the clarification of study phases. As stated in Section 3.2.2.1 of the E8 guideline, the selection of subjects treated in the later stages of the development process, i.e., in phase III confirmatory studies, should be broadened to reflect the target population. Another important aspect of conducting a phase III comparative study is to ascertain the suitable patient population in relation to the source and immunogenicity of the cells to be used for transplantation, the biosafety of medium components, and the route of administration of therapy [2].

Variation in the effectiveness of therapies is inevitably encountered in clinical trials. Although some of the reasons for this variation are mentioned above, there are many other considerations. For example, in drug trials involving small molecules or antibodies, drug quality and administration procedures are generally highly consistent. However, in the case of regenerative therapies with autologous stem cells, functional and qualitative differences in stem cell preparations may still exist, even if stringent quality control measures are in place [3]. Another issue is the source of stem cells [4*0]. Embryonic stem cells, induced pluripotent stem cells (iPSCs), and various somatic stem cells, including mesenchymal stem cells (MSCs) from many organs, such as bone marrow, umbilical cord, and adipose tissue, have been assessed in human clinical trials. The factors underlying the variation in efficacy and safety of stem cell transplantation are not well understood. The enrollment of sufficient numbers of trial participants from diverse backgrounds is clearly key to addressing this issue $[4 \cdot \bullet]$.

\section{Considerations of Diversity in Basic Studies}

One characteristic of regenerative medicine is that cell sources are obtained from human in most of the cases. MSCs show self-renewal capacity, multi-lineage differentiation potential, and ability to migrate toward sites of inflammation or injury and have been investigated for autologous or allogeneic use. In a recent study involving the prospective purification of vascular presumptive MSCs from human adipose tissue, the frequency and yield of perivascular stem cells (PSCs) and the correlation with demographic data were assessed by using samples obtained from 131 healthy donors [5•]. The number and viability of PSCs were largely unaffected by demographic parameters, such as donor age, sex, or body mass index. However, the quality and the consistency of processed cells were significantly influenced by procedure-based variables. Although demographic parameters were reported not to influence the results in this study, this conclusion should be interpreted with caution, because the data were not obtained from a clinical trial and detailed demographic data were not presented and could not be readily accessed. The application of ethnically diverse iPSCs for drug development has been attempted [6, 7], and iPSC banks for pharmacogenomic studies have been established by using cells from a broad range of donors [7]. The rationale behind these studies is based on the concept that genetic differences affect the responses of iPSCs to drugs and other therapeutic interventions. Although these studies are not clinical trials, the concepts behind them highlight the importance of assessing and understanding the different responses of iPSCs used in allogeneic treatment.

\section{Elderly Patients}

Age is an important demographic parameter impacting clinical trials, exemplified by ICH-E7, "STUDIES IN SUPPORT OF SPECIAL POPULATIONS: GERIATRICS.” In regenerative medicine, both aging of recipients and aging of stem cells should be considered concurrently. Although a discussion of HSC transplantation is outside the scope of this article, age-related changes in HSCs, such as increases in genomic instability, oxidative stress, protein aggregation, and inflammation and decreases in DNA repair ability, metabolic biogenesis, epigenetic modulation, and telomere function [8], may be representative of changes affecting other stem cell types. Long-term culture of MSCs in vitro resulted in reductions in growth kinetics, telomere length, and differentiation capability 
[9], suggesting that the enrollment of aged patients in stem cell clinical trials should be carefully considered. Similarly in animal models, rat studies of ischemic myocardium revealed that bone marrow stromal cells obtained from younger donors were more effective for myocardial regeneration compared with those from older donors [10]. Various preclinical approaches have been used to reinvigorate aged stem cells, including the addition of sildenafil or histone deacetylace inhibitors at preconditioning or augmentation of culture media with growth factors, cytokines, or chemokines, including membrane protein Notch or Pim-1 kinase during processing, and reported to be hopeful. However, these methods have not been tested in clinical trials, and efficacies in human have not been clarified [11-15]. Interestingly, treatment of patients with knee joint chondral defects by inoculation of autologous MSCs embedded in a hyaluronan-based scaffold showed that efficacies were mainly affected by the size and number knee joint chondral defects, rather than the age of the patient [16•]. Indeed, the functional outcomes of patients older than 45 years were comparable to younger patients at final follow-up. Adding to the age of patients, "age" of the source of cells is the issue to be considered. Umbilical cord-derived MSCs showed a longer culture, a large scale expansion, a retardation of senescence, and a higher anti-inflammation effect than adult bone marrow and adipose tissue-derived MSCs in preclinical study [17]. Fetal bone marrow-derived MSCs and umbilical cord-derived MSCs presented biological advantages as compared to adult adipose tissue-derived MSCs in preclinical study, and umbilical cord-derived MSCs have a gene expression pattern similar to adult adipose tissue-derived MSC but not fetal bone marrow-derived MSCs [18]. Because most clinical trials by using MSCs lack long-term follow-up and the number of subjects is often insufficient, the question of how patient age and the difference source, such as fatal tissue derived or adult tissue derived, affect the long-term results of stem cell therapies remains to be resolved [19••].

\section{The Influence of Gender}

According to ICH-E3, "STRUCTURE AND CONTENT OF CLINICAL STUDY REPORTS," it may also be useful to include demographic data including gender into clinical study reports, and patient gender is a critical variable usually including into clinical trial demographic variable. In the review on anesthesiology drugs, gender differences, such as decreased effect of propofol and increased effect of morphine in females, were shown [20]. Tajiri et al. reviewed the characteristics of gender-specific stem cells [21••]. Testis-derived Sertoli cells in rat study showed immunosuppressive effects and secreted growth and trophic factors inducing functional recovery of animals transplanted with fetal dopaminergic cells [20]. From the female perspective, menstrual blood stem cells display angiogenic effects, proliferative capability, and pluripotency. Although these cells are gender specific in nature, sex-specific differences in somatic tissue-derived cells have been described [22, 23]. Female bone marrow-derived MSCs exhibited increased expression of IFN- $\gamma$ R 1 and IL- $6 \beta$ and were more potent in T cell proliferation suppression compared with male MSCs [22]. High clonogenic bone marrowderived MSCs from younger females were smaller, divided more rapidly, and were more frequent than MSCs isolated from older females [22]. A study comparing primary osteoblast cell lines derived from male and female sagittal and metopic craniosynostosis patients revealed reduced proliferation in the male cell lines [23]. Although gender differences have been observed in preclinical studies, it remains to be seen whether these differences will be reproduced in clinical trials.

\section{The Difficulties of Evaluating the Efficacy of Stem Cell Therapies}

Stem cell therapies aim to replace defective and/or functionally compromised organs with new tissue to provide a stable long-term curative outcome. In clinical trials involving transplanted tissues, rather than individual cells, both patient factors (i.e., degree of functional deterioration and histological/anatomical deformity) and graft characteristics (i.e., histological structure and function) should be considered as inclusion criteria. In relation to patient factors, it is important that inclusion decisions are based on robust criteria. For example, Jacobson et al. reported the most optimal retinal structural measurement methods for stem cell clinical trials for retinal degeneration [24]. They undertook optical coherence tomography and autofluorescence imaging of retinitis pigmentosa and macular degeneration patients and found that retinitis pigmentosa patients had limited visual fields and maculopathy patients had central scotomas with retained peripheral function. These differences were consistent with histological structure analyses $[24,25 \cdot, 26]$. It was suggested that the differences in the effectiveness of the imaging approaches may be useful for determining inclusion/exclusion criteria for stem cell therapies for retinal degeneration. These types of early studies are critical for determining both optimal inclusion criteria and whether clinical trials addressing the target disease or condition should be limited or expanded.

Chondral defects of the knee is a disease for which many treatment options have been approved and many clinical trials have been conducted [16•, 27, 28]. Reparative fibrocartilage has inferior biological and biomechanical properties compared with hyaline cartilage and is associated with increased post-operative recurrence of osteoarthritis. Although many attempts have been made to improve hyaline cartilage processing and transplantation methods [16•,27, 28], it is unclear whether other factors, such as patient and/or donor 
characteristics, affect therapeutic outcomes. A comparison of patients with different conditions and backgrounds in appropriately designed clinical trials may help answer this question, as shown in the retinal disease study mentioned previously, although it is difficult to compare the results of histological data conducted in independent laboratories.

\section{Current Schema of Stem Cell Therapies Registered in 2015}

The enrollment of a large number of patients in a randomized clinical trial is an appropriate way to avoid patient selection bias and comprehensively address the effectiveness of treatment after approval. In stem cell clinical trials, enrolling a large number of patients is often difficult, even in phase III studies, because the target diseases are usually rare and/or the preparation of large numbers of cells is technically difficult, especially for autologous cell-based products. These factors may significantly compromise patient diversity in clinical trials. Table 1 shows the schemas for the development of stem cell therapies that were submitted to "ClinicalTrials.gov" (https://clinicaltrials.gov/) in 2015. Clinical trials containing the following words were selected: "phase III," "stem cell," "regenerative," "stem cell," "mesenchymal," "msc," and "es cell." Patient numbers in these clinical trials ranged from 2 to 330 , and three of nine studies were single-arm trials, although researchers designated their studies as phase III. Five of the nine studies were described as "phase II/III," which is a design that has been used to shorten the time of clinical development, especially in clinical trials for life-threatening diseases. The approach also reduces the number of subjects required, especially for rare diseases, although there are special rules regarding the subject number for the phase III portions of combination trials $[29,30]$. As shown in Table 1, the enrollment of relatively small numbers of patients in consecutive clinical trials is clearly a concern when considering the importance of patient diversity and the reduced power associated with the statistical analysis of small numbers of subjects.

\section{Fast-Track Approval in Japan}

Faster approval based on a smaller number of subjects in earlier clinical trials is critical, especially for patients with lifethreatening diseases. The US Food and Drug Administration (FDA) developed an "accelerated approval" process, whereby drugs and biologics can be approved based on surrogate endpoints considered to be closely related to the true endpoint, such as response rate is closely related to survival benefit in cancer patients [31]. Under this system, a phase IV study must be done to prove efficacy after accelerated approval. The broader selection of patients that can be enrolled in phase IV trials may enable the analyses of nonprotocol prespecified factors, including alternative biomarkers. In Japan, the Pharmaceuticals, Medical Devices, and Other Therapeutic Products Act was revised and enforced in 2014, and conditional and time-limited approval for regenerative products was introduced to accelerate the development of regenerative medical products [32•]. Regenerative medical products had been classified as either drugs or medical devices until the revision. This system enables certain regenerative medical products to be approved, if the safety of the products and the likely efficacy are demonstrated through small-scale clinical trials [5•].

Table 1 Schema of phase III studies in "ClinicalTrials.gov" received in 2015

\begin{tabular}{|c|c|c|c|c|}
\hline Disease & Cell product & Phase & $\begin{array}{l}\text { Treatment: study arm (S), } \\
\text { control arm }(\mathrm{C})\end{array}$ & $\begin{array}{l}\text { Number of } \\
\text { patients }\end{array}$ \\
\hline Diabetic peripheral neuropathy & Auto, BM MSC & Phase II/III & $\mathrm{S}$ & 10 \\
\hline Tibia nonunion & Auto, BM MSC & Phase II/III & $\begin{array}{l}\text { MSC with platelet lysate }(\mathrm{S}), \\
\text { placebo }(\mathrm{C})\end{array}$ & 60 \\
\hline Spinal cord injury & $\mathrm{UB}, \mathrm{MSC}$ & Phase III & $\mathrm{S}$ & 44 \\
\hline Chronic discogenic lumbar back pain & Allo, MSC & Phase III & $\begin{array}{l}\text { MSC or MSC with hyaluronic } \\
\text { acid (S: two arms), placebo (C) }\end{array}$ & 330 \\
\hline Male stress urinary incontinence & Auto, adipose-derived regenerative cells & Phase III & $\mathrm{S}$ & 45 \\
\hline Degenerative arthritis & Auto, adipose, MSC & Phase II/III & MSC (S), saline (C) & 120 \\
\hline Critical limb ischemia & $\begin{array}{l}\text { Auto, peripheral blood endothelial } \\
\text { progenitor cells }\end{array}$ & Phase II/III & $\begin{array}{l}\text { Peripheral blood endothelial } \\
\text { progenitor cells (S), BM } \\
\text { mononuclear cells }(\mathrm{C})\end{array}$ & 38 \\
\hline Myocardial infarction & Allo, low-oxygen BM MSC & Phase III & $\operatorname{MSC}(\mathrm{S})$, placebo $(\mathrm{C})$ & 50 \\
\hline Crohn's disease & $\mathrm{MSC}^{\mathrm{a}}$ & Phase II/III & $\begin{array}{l}3 \times 10^{6} / \mathrm{kg} \text { or } 6 \times 10^{6} / \mathrm{kg} \text { MSC } \\
\quad(\mathrm{S}: \text { two arms), normal saline }(\mathrm{C})\end{array}$ & 3 \\
\hline
\end{tabular}

Auto autologous, MSC mesenchymal stem cell, BM bone marrow, UB umbilical cord, Allo allogeneic

a Insufficient information on "ClinicalTrials.gov" 
After approval through this system, the marketing authorization holder must conduct post-marketing surveillance to assess the efficacy and safety in an appropriately large number of patients and submit any additional safety and efficacy data to the Pharmaceuticals and Medical Devices Agency (PMDA) within 7 years. The scheme of conditional and time-limited approval for regenerative products is shown in Fig. 1. Based on the post-marketing surveillance data, a decision will be made on whether conditional approval will be allowed to expire or regular approval will be granted. HeartSheet, a skeletal muscle-derived myoblast tissue, was the first therapy approved for patients with severe heart failure under the conditional and time-limited scheme. The pivotal study for HeartSheet was a phase II trial involving seven patients [33•]. A post-marketing study comparing patients treated with HeartSheet $(n=60)$ with an external concurrent cohort ( $n=120)$ utilizing statistical matching was mandated by the PMDA. This post-marketing study was funded by the patients' health insurance provider and not by the sponsor. This ensures that the financial burden of conducting patient surveillance or clinical trials is decreased considerably for the sponsor and sufficient information on patients' backgrounds and efficacy and/or safety data will be obtained. Although there are some concerns that this scheme might facilitate approval based on the data obtained from only a small number of patients in earlier clinical trials, the conduct of appropriately designed post-marketing surveillance may provide a good opportunity to study the influences of patient diversity on the outcomes of clinical trials [34].

\section{Biomarker Study}

In the USA, biomarker endpoints have been incorporated into FDA approvals for rare disease therapies [35]. The identification of qualifying biomarkers is necessary for detecting predictors of favorable or unfavorable therapeutic response. Many attempts to translate the information on biomarkers and genetic profiling obtained from laboratory and animal studies into clinical practice have been made in stem cell research $[3,5 \cdot 36]$. TNF- $\alpha$, TNF receptor- 1 , TNF receptor- 2 , or adiponectin are expected to be putative biomarkers of monitoring anti-inflammatory effects of MSCs for patients with diabetic kidney disease [37]. Cell surface proteome of cultured adipose MSCs $(n=5)$ was conducted [38]. Known MSC markers, such as CD29, CD44, CD73, CD90, and CD105, were strongly expressed. However, entirely different level of expression of CD4, CD70, CD56, CD30, and CD41a was also detected. Adding to these markers, different level of expression of 66 markers was observed. The study on the therapeutic effects based on these differences is the future problem; it would be useful to pay attention to the diverse characteristics of MSCs and stock MSCs for the future analysis. Conclusively, for the promotion of biomarker study, analyses of cells (both at the time of collection and after processing) and collection of information on patient before and after regenerative medicine are required.

\section{Conclusion}

The translation of stem cell therapies to the clinic still faces many hurdles, including the selection of appropriate numbers of suitable patients and controls for clinical trials. In addition, a greater understanding of the impact of patient diversitygenetic, ethnic, environmental-is required to comprehensively address the efficacy and safety of stem cell interventions for individualized therapy. Approaches to solve this problem include stringent efforts to recruit patients from diverse backgrounds and the long-term follow-up of a larger number of patients, both of which represent significant challenges for the design of future stem cell clinical trials.
Fig. 1 Conditional and timelimited approval for regenerative products in Japan. Revised figure announced by the Ministry of Health, Labor, and Welfare. (http://www.kantei.go. jp/jp/singi/tiiki/kokusentoc wg/hearing_s/150313shiryou0101.pdf)

\section{【Old pathway】}

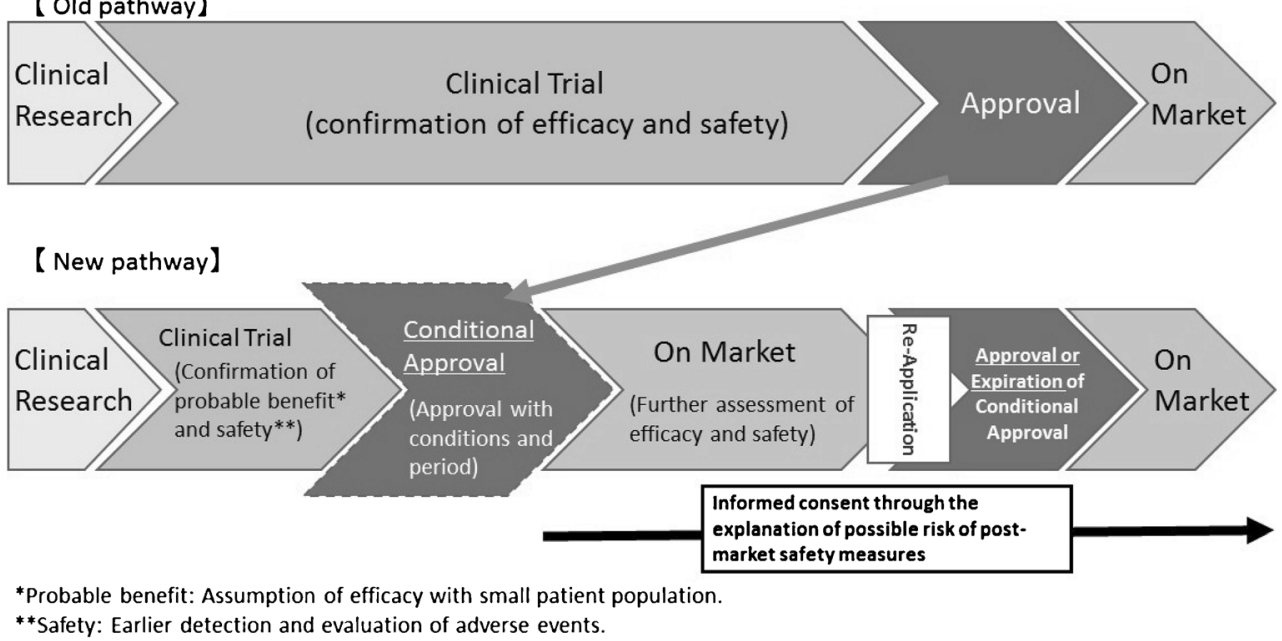




\section{Compliance with Ethical Standards}

Conflict of Interest Fumitaka Nagamura is a member of the data monitoring committee of oncology clinical trials conducted by Yakult Co. But, he declares that he has no conflict of interest for this article.

Human and Animal Rights and Informed Consent This article does not contain any studies with human or animal subjects performed by the author.

\section{References}

Papers of particular interest, published recently, have been highlighted as:

- Of importance

•- Of major importance

1. Rochon PA, Berger PB, Gordon M. The evolution of clinical trials: inclusion and representation. CMAJ. 1998;159:1373-4.

2. Bernardo ME, Fibbe WE. Safety and efficacy of mesenchymal stromal cell therapy in autoimmune disorders. Ann N Y Acad Sci. 2012;1266:107-17.

3. Kagami $H$, Agata $H$, Inoue $M$, et al. The use of bone marrow stromal cells (bone marrow-derived multipotent mesenchymal stromal cells) for alveolar bone tissue engineering: basic science to clinical translation. Tissue Eng Part B Rev. 2014;20:229-32.

4.• Blum HE. Advances in individualized and regenerative medicine. Adv Med Sci. 2014;59:7-12. This review provides the information on the characteristics and history of development of iPS cells, ES cells and MSCs.

5. West CC, Hardy WR, Murray IR, et al. Prospective purification of perivascular presumptive mesenchymal stem cells from human adipose tissue: process optimization and cell population metrics across a large cohort of diverse demographics. Stem Cell Res Ther. 2016;7:47. This study provides the influence of patient age, gender, BMI, the storage time of the tissue or procedure based variables on prospective purification of perivascular presumptive MSCs from adipose tissue.

6. Fakunle ES, Loring JF. Ethnically diverse pluripotent stem cells for drug development. Trends Mol Med. 2012;18:709-16.

7. Takayama K, Morisaki Y, Kuno S, et al. Prediction of interindividual differences in hepatic functions and drug sensitivity by using human iPS-derived hepatocytes. Proc Natl Acad Sci U S A. 2014;111:16772-7.

8. Van Zant G, Liang Y. Concise review: hematopoietic stem cell aging, life span, and transplantation. Stem Cells Transl Med. 2012;1:651-7.

9. Bonab MM, Alimoghaddam K, Talebian F, et al. Aging of mesenchymal stem cell in vitro. BMC Cell Biol. 2006;7:14.

10. Zhang H, Fazel S, Tian H, et al. Increasing donor age adversely impacts beneficial effects of bone marrow but not smooth muscle myocardial cell therapy. Am J Physiol Heart Circ Physiol. 2005;289:H2089-96.

11. Khatiwala R, Cai C. Strategies to enhance the effectiveness of adult stem cell therapy for ischemic heart diseases affecting the elderly patients. Stem Cell Rev. 2016;12:214-23.

12. Hoke NN, Salloum FN, Kass DA, Das A, Kukreja RC. Preconditioning by phosphodiesterase- 5 inhibition improves therapeutic efficacy of adipose-derived stem cells following myocardial infarction in mice. Stem Cells. 2012;30:326-35.

13. Zhang L, Qin X, Zhao Y, et al. Inhibition of histone deacetylases preserves myocardial performance and prevents cardiac remodeling through stimulation of endogenous angiomyogenesis. J Pharmacol Exp Ther. 2012;341:285-93.

14. Conboy IM, Conboy MJ, Wagers AJ, et al. Rejuvenation of aged progenitor cells by exposure to a young systemic environment. Nature. 2005;433:760-4.

15. Mohsin S, Khan M, Nguyen J, et al. Rejuvenation of human cardiac progenitor cells with Pim-1 kinase. Circ Res. 2013;113:1169-79.

16. Gobbi A, Scotti C, Karnatzikos G, et al. One-step surgery with multipotent stem cells and Hyaluronan-based scaffold for the treatment of full-thickness chondral defects of the knee in patients older than 45 years. Knee Surg Sports Traumatol Arthrosc. 2016. Published online: 14 January 2016. This study provides the information that patient age was not related to the clinical outcome in regenerative medicine for the treatment of chondral defects of the knee in patients older than $\mathbf{4 5}$ years.

17. Jin HJ, Bae YK, Kim M, et al. Comparative analysis of human mesenchymal stem cells from bone marrow, adipose tissue, and umbilical cord blood as sources of cell therapy. Int J Mol Sci. 2013;14:17986-8001.

18. Wang Q, Yang Q, Wang Z, et al. Comparative analysis of human mesenchymal stem cells from fetal-bone marrow, adipose tissue, and Warton's jelly as sources of cell immunomodulatory therapy. Hum Vaccin Immunother. 2016;12:85-96.

19.• Schimke MM, Marozin S, Lepperdinger G. Patient-specific age: the other side of the coin in advanced mesenchymal stem cell therapy. Front Physiol. 2015;6:362. This review shows the influences of patient age on functions of MSCs.

20. Pleym H, Spigset O, Kharasch ED, et al. Gender differences in drug effects: implications for anesthesiologists. Acta Anaesthesiol Scand. 2003;47:241-59.

21.• Tajiri N, Duncan K, Borlongan MC, et al. Adult stem cell transplantation: is gender a factor in stemness? Int J Mol Sci. 2014;15: 15225-43. This study provides the potential of gender-specific stem cells as candidate cell source for regenerative medicine for patients with stroke.

22. Siegel G, Kluba T, Hermanutz-Klein U, et al. Phenotype, donor age and gender affect function of human bone marrow-derived mesenchymal stromal cells. BMC Med. 2013;11:146.

23. Park SS, Beyer RP, Smyth MD, et al. Osteoblast differentiation profiles define sex specific gene expression patterns in craniosynostosis. Bone. 2015;76:169-76.

24. Jacobson SG, Matsui R, Sumaroka A, Cideciyan AV. Retinal structure measurements as inclusion criteria for stem cell-based therapies of retinal degenerations. Invest Ophthalmol Vis Sci. 2016;57. ORSFn1-9

25. Nazari H, Zhang L, Zhu D, et al. Stem cell based therapies for agerelated macular degeneration: the promises and the challenges. Prog Retin Eye Res. 2015;48:1-39. This study provides the information to apply stem cell therapy for age-related macular degeneration.

26. Garcia JM, Mendonça L, Brant R, et al. Stem cell therapy for retinal diseases. World J Stem Cells. 2015;7:160-4.

27. Kon E, Gobbi A, Filardo G, et al. Arthroscopic second-generation autologous chondrocyte implantation compared with microfracture for chondral lesions of the knee: prospective nonrandomized study at 5 years. Am J Sports Med. 2009;37:33-41.

28. Gobbi A, Kon E, Berruto M, et al. Patellofemoral full-thickness chondral defects treated with Hyalograft-C: a clinical, arthroscopic, and histologic review. Am J Sports Med. 2006;34:1763-73.

29. Liu Q, Pledger GW. Phase 2 and 3 combination designs to accelerate drug development. J Am Stat Assoc. 2005;100:493-502.

30. Liu Q. Wiley Encyclopedia of Clinical Trials. Phase 2/3 trials. p. 1-12. 
31. Johnson JR, Ning YM, Farrell A, et al. Accelerated approval of oncology products: the food and drug administration experience. $\mathrm{J}$ Natl Cancer Inst. 2011;103:636-44.

32. Konishi A, Sakushima K, Isobe S, Sato D. First approval of regenerative medical products under the PMD act in Japan. Cell Stem Cell. 2016;18:434-5. This report shows the approved regenerative medical products under the Pharmaceuticals, Medical Devices, and Other Therapeutic Products Act in Japan.

33. Fujita Y, Kawamoto A. Regenerative medicine legislation in Japan for fast provision of cell therapy products. Clin Pharmacol Ther. 2016;99: 26-9. This report shows the scheme of conditioning and timelimited approval for regenerative medical products in Japan.

34. Cyranoski D. Japan to offer fast-track approval path for stem cell therapies. Nat Med. 2013;19:510.
35. Kakkis ED, O’Donovan M, Cox G, et al. Recommendations for the development of rare disease drugs using the accelerated approval pathway and for qualifying biomarkers as primary endpoints. Orphanet J Rare Dis. 2015;10:16.

36. Mancini M, Zazzara M, Zattoni F. Stem cells, biomarkers and genetic profiling: approaching future challenges in urology. Urologia. 2016;83:4-13.

37. Griffin TP, Martin WP, Islam N, et al. The promise of mesenchymal stem cell therapy for diabetic kidney disease. Curr Diab Rep. 2016;16:42.

38. Donnenberg AD, Meyer EM, Rubin JP, Donnenberg VS. The cellsurface proteome of cultured adipose stromal cells. Cytometry A. 2015;87:665-74. 